\title{
Optimal Control of fed batch bioreactor
}

\author{
Durgesh Bonde and Dr. Satish Inamdar
}

\begin{abstract}
In this paper, we will consider the problem of optimal control of a fed batch reactor. Our objective is to simulate the fed batch reactor under specified conditions in order to find an optimal control policy. Thus, for any specified initial conditions and parameter values, the optimal policy for reactor operation can be obtained from simulation. We have an example system of nosiheptide and used the gradient method to find the optimal policy. Although the convergence is slow, an optimal solution is obtained and various plots are prepared that illustrate the applicability of the method well.
\end{abstract}

Index Terms - Fed batch reactor; optimal control; Pontryagin's minimum principle; Gradient method.

\section{INTRODUCTION}

Batch reactors are commonly used in the chemical industry specially to produce food products, fine chemicals, and specialty chemical products[1]. The variations during operation can cause concern about the quality and yield of the final chemical product and hence an optimal policy for the control of the operation is necessary[2]. Various measures are brought into focus in order to search for causes of variations and those termed batch-to-batch variations. The dynamic optimization problem points to following a minimum energy path to attain optimum results from a batch run[3]. Examples are reaching equilibrium point as saturation limit in dissolved oxygen level during fed batch bioreactor operation and setting progression of reaction temperature that will guarantee maximum conversion and yield from catalytic reactions. In sum forming an optimal operation policy for batch reactor operations is an important consideration while scheduling tasks in the batch process industry.

Now we will present a brief literature review. Reference [4], reports work on optimal closed-loop control of a batch polymerization reactor. They presented computer optimal closed-loop control policies which were derived and applied to the simulation of a batch reactor. A multivariable feedback controller could track the nominal open-loop optimal state trajectories although disturbances in the process were present. An analysis based on distributional and worst-case scenarios of the open and closed-loop control of batch processes concluded that the fundamental feature of batch process optimization is that the expected performance is expressed as a function of final states[5].

Manuscript revised on May 29, 2021 and published on June 10, 2021

Durgesh Bonde, Masters Student in Chemical Engineering at

Northeastern University, Boston

Dr. Satish Inamdar, Professor (Retired), (Corresponding author)

Email: satishinamdar2810@gmail.com
Hence the objective function for optimization will contain the final product and state variable specification. Stacked neural networks have been used to enhance the model generalization ability within confidence bounds to predict performance from process operational data[6]. It was observed from the simulations that neural networks can improve process performance from batch to batch even though model plant mismatches and unknown disturbances were present.

Dynamic optimization of fed batch reactor has been extensively studied, which often involves finding an optimal feed profile that maximizes either the yield of the desired products or includes optimization of objective function [7][9].

Since, the operation of a fed-batch reactor is a complex process, involving high-value materials or reactants, the control and optimization of the processes become an important practical issue. Optimal control in such cases is generally a multi-objective optimal problem, weighted sum approach is also used[7].

Now we will present a motivating example.

\section{Motivating EXAMPLE}

We consider the fed-batch reactor model for nosiheptide reactor[1]. Nosiheptide is produced as a metabolite by microorganisms and is a sulfur-containing peptide antibiotic[8],[9]. The model equations are given here.

$$
\begin{aligned}
& \frac{d X}{d t}=f_{1}=\left(\mu_{g}-\mu_{d}-\frac{F}{V}\right) X \\
& \mu_{g}=\frac{A_{g} e^{-\frac{E g}{R T}}}{1+\frac{K_{1}}{10^{-p H}}+\frac{10^{-p H}}{K_{2}}} \frac{S}{K_{S} X+S} \frac{C O}{K_{O} X+C O}\left(1-\frac{X}{X_{M A X}}\right) \\
& \mu_{d}=A_{d} e^{-\frac{E_{d}}{R T}}\left(1-\frac{C O}{K_{d}+C O}\right) \\
& \frac{d S}{d t}=f_{2}=-m_{S} X-\frac{1}{Y_{X / S}} \frac{d X}{d t}-\frac{1}{Y_{P / S}} \frac{d P}{d t}-\frac{F}{V} X \\
& \frac{d P}{d t}=f_{3}=\beta X-K_{h} P-\frac{F}{V} X \\
& \beta=\frac{\mu_{P} S}{K_{P}+S+\frac{S^{2}}{K_{I}}} \\
& \frac{d V}{d t}=f_{4}=F \\
& \frac{d C O}{d t}=f_{5}=K_{L a}\left(C O^{*}-C O\right)-m_{O} X-\frac{1}{Y_{X / O}} \frac{d X}{d t}-\frac{1}{Y_{P / o}} \frac{d P}{d t}-\frac{F}{V} C O(5) \\
& C O^{*}=0.037 \mathrm{gL}^{-1}\left(T=29^{0} \mathrm{C}\right) \\
& K_{L a}=0.1322 \frac{d^{0.56} n^{0.18} P^{0.36} Q^{0.3992}}{D V^{0.4}}
\end{aligned}
$$

There are five state variables which are biomass concentration $(\mathrm{X})$ in the fermentation broth, substrate concentration (S), microbial metabolite (i.e., nosiheptide) 
concentration $(\mathrm{P})$, volume of media in a bioreactor $(\mathrm{V})$, dissolved oxygen level in fermentation broth during bioreaction (CO). The substrate is used as nutrients for cell growth, metabolite production, and maintaining bacteria culture activity. The terms $m_{O}$ and $m_{S}$ represent the maintenance coefficient of dissolved oxygen and substrate respectively.

The main objective of this work is to simulate a fed batch reactor for the production of nosiheptide and explicitly find an optimal control policy. The policy is chosen such that the reactor operation tracks four state variables namely, dissolved oxygen concentration, biomass concentration, substrate consumption, and product concentration by manipulating the feed rate $(\mathrm{F})$ over the reactor operation time. This is achieved by choosing an objective function and minimizing the function as an optimal control problem. The deviation of the state variables is weighted along with a penalizing function for excessive control moves.

\section{OPTIMAL CONTROL}

We will give a summary of the optimal control theory as being applied to the fed batch reactor. The performance index or objective function is defined as

$$
\begin{gathered}
J=\int_{t_{o}}^{t_{f}} \eta\left(C O^{*}-C O[t]\right)^{2}+\zeta(P f-P[t])^{2}+\epsilon(s f-S[t])^{2}+ \\
\alpha\left(u R e f-u[t]^{2}\right)+\gamma\left(1-\frac{X}{X_{M A X}}\right)^{2}
\end{gathered}
$$

The above objective function is to be minimized using the gradient method, so to track saturated dissolved concentration in the broth $\left(\mathrm{CO}^{*}\right)$, maximizing biomass concentration and consumption of substrate, along with tracking an arbitrary set point of product concentration to maximize product formation and introducing a penalizing function for controlling excessive control moves.

The Hamiltonian for the optimal control problem is obtained using (1)-(6), resulting in

$H=\eta\left(C O^{*}-C O[t]\right)^{2}+\zeta(P f-P[t])^{2}+\epsilon(s f-S[t])^{2}+$

$\alpha\left(u R e f-u[t]^{2}\right)+\gamma\left(1-\frac{X}{X_{M A X}}\right)^{2}+\sum_{i=1}^{5} \lambda_{i} f_{i}$

where $f_{i}, i=1,2, \ldots, 5$ are rhs of (1) - (5). And $\lambda_{i}, i=1,2, \ldots, 5$ are costate variables.

Applying Pontryagin's minimum principle to (7), we obtain

$$
\begin{aligned}
& \frac{\partial H}{\partial u}=0 \\
& \frac{\partial \lambda^{T}}{\partial t}=\frac{\partial H}{\partial z}
\end{aligned}
$$

Here, $z \equiv\{X, S, P, V, C O\}$, where $z$ is a state vector. First, we obtain $u^{*}$ by solving for (8) and later see that (9) form costate differential equations.

\section{NUMERICAL COMPUTATIONS}

The gradient method is explained here. First, we assume a starting value for $u[t], t_{0} \leq t \leq t_{f}$. Integrate system dynamics to find out state vector as a time history. In a symbolic manipulator, the solution is expressed as an interpolating polynomial. These are needed to integrate costate equations. Now, integrate costate or adjoint equations to find out $\lambda_{i}, i=1,2, \ldots, 5$ in the backward direction. The $u[t]$ is updated using a relation as

$$
\Delta u[t]=-W[t]^{-1}\left(\frac{\partial L}{\partial u}+\lambda^{T} \frac{\partial f}{\partial u}\right)
$$

where $\mathrm{W}[\mathrm{t}]$ is a weighing matrix, the $\mathrm{L}$ is the Lagrangian[10],[11]. And $f=\left\{f_{1}, \ldots, f_{5}\right\}$. Now, continue with the iterative procedure until $H[u[t]]$ goes to zero. It is observed that the stability is improved and convergence is slow to terminate the run.

We use the same parameter values as given in the system of nosiheptide for the simulation experiment[1]. However, our initial conditions differ; these are given as, $X[0]=$ $0.05, S[0]=12.0, P[0]=0.0, V[0]=60.0, C O[0]=0.037$.

\section{RESULTS AND DISCUSSION}

Upon execution of the above procedure, and solving the optimization problem by gradient method, we obtain a profile of $u[t]$ versus $t$ that gives an optimal feed policy. Moreover, for understanding the system and the effect of various parameters on the optimal control policy, a parametric study has been carried out. The result section is divided into two parts. In the first part, we vary two parameters, initial biomass concentration, and $P f$, which is an arbitrary point used for maximizing product concentration, and their effect on optimal feed control is studied. In the second part, we consider various combinations of terms in the objective function and their respective weightage and compute the optimal control profiles. These are seen to reveal interesting results that are discussed.

TABLE I: First Experiment. Comparison of options for optimal control

\begin{tabular}{ccccccc}
\multicolumn{7}{c}{ policy } \\
\hline \hline Case & $\mathrm{X}_{\mathrm{a}}$ & $\alpha$ & $\eta$ & $\boldsymbol{\gamma}$ & $\boldsymbol{\epsilon}$ & $\zeta$ \\
\hline 1 & 0.005 & 0 & 0.4 & 0.00085 & 0.00093 & 0 \\
2 & 0.1 & 2.4 & 0 & 0.00085 & 0.00093 & 0.0082 \\
3 & 0.2 & 2.4 & 0.4 & 0 & 0.00093 & 0 \\
4 & 0.4 & 2.4 & 0.4 & 0.00085 & 0 & 0.00082 \\
5 & 0.7 & 2.4 & 0.4 & 0.0085 & 0.00093 & 0 \\
\hline \hline
\end{tabular}

In the first numerical experiment performed, we have varied the values of initial biomass concentration and $P f$ as mentioned in Table I. A gradient method is used to solve the optimal control problem keeping the other parameters constant for all runs. Fig. 1 represents the optimal control feed policy obtained for the three cases. There is a decrease observed in the feed rate at a very early stage, pointing to the fact that there is an initial charge of biomass in the reactor. It is observed that more substrate is added as control action required when initial biomass concentration is increased. 
International Journal of Research in Advent Technology, Vol.9, No.5, May 2021

E-ISSN: 2321-9637

Available online at www.ijrat.org

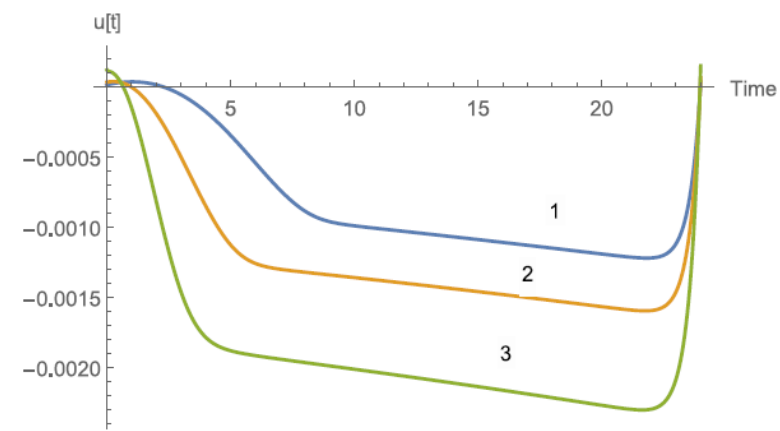

Fig.1: First Numerical Experiment. Feed rate control

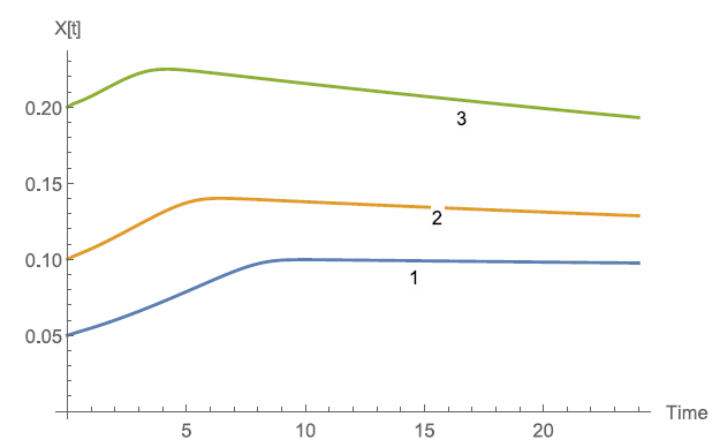

Fig.2: First Numerical Experiment. Biomass growth

Fig. 2 depicts the biomass concentration with respect to batch operation time. With the increase in time, biomass growth occurs due to the consumption of substrate to produce the product. The significant observation is that when a higher initial concentration of biomass is used, the death rates come into force much more significantly than for lower biomass concentrations. This explains the decrease in biomass concentrations during the operation time.

The substrate concentrations for all three cases are shown in Fig. 3. It is obvious that the substrate concentration with time will decrease as the biomass formed consumes the substrate to form the product. The substrate action considers three terms that emphasize providing nutrients or cell growth, producing metabolites, and maintaining culture activity as expressed in (3). The substrate concentration falls more rapidly for case 3 with a higher initial biomass concentration. Similarly, the rate of substrate consumption reduces with an increase in initial biomass concentration.

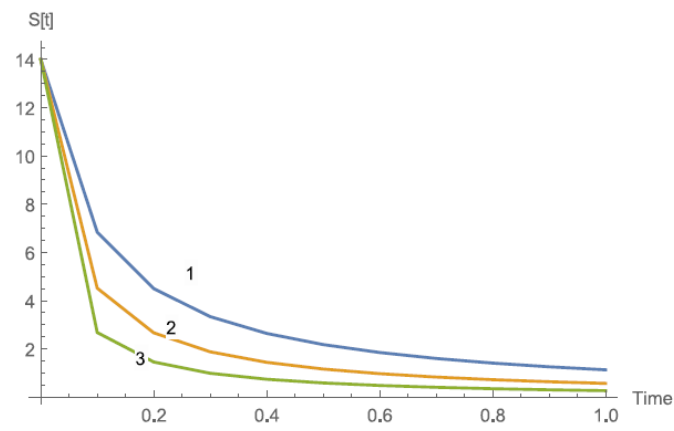

Figure 3: First numerical experiment. Substrate consumption

The product concentration is shown in Fig. 4. Here it is observed that the product concentration stabilizes rapidly for case 3 when compared with the other cases. For case 1 due to low initial biomass concentration, the bioreaction takes a slower path for the product formation. Here, we need to note that, the $P f$ term is not the setpoint to achieve, but is arbitrarily assumed for maximizing the product concentration. It is observed that, even though we change the values of $P f$, there is no significant change in the result of the product concentration.

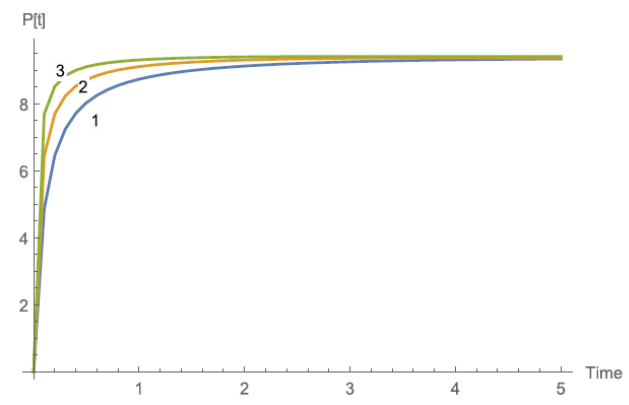

Figure 4: First numerical experiment. Product formed

A higher initial biomass loading is observed to have higher rates of information of product; however, it requires higher control of feed rate as is evident from Fig. 1. The case with low initial biomass concentration may be slow, but requires less control over the optimal control feed policy.

The objective function plays a vital role in determining the optimal control policy. The objective function in this work comprises weighted deviations, to track the values required. The change in weights can be decisive in opting for a control

TABLE II: Second experiment. Comparison of options for the objective function

\begin{tabular}{|c|c|c|c|c|c|c|c|c|c|c|c|}
\hline Case & $t_{o}$ & $t_{f}$ & $\alpha$ & $\eta$ & $\gamma$ & $\epsilon$ & $\zeta$ & $s_{f}$ & $V_{i}$ & $X_{o}$ & $P f$ \\
\hline 1 & 0 & 24 & 0.4 & 0.4 & 0.00005 & 0.00003 & 0.00002 & 14 & 60 & 0.05 & 7.5 \\
\hline 2 & 0 & 24 & 0.4 & 0.4 & 0.00005 & 0.00003 & 0.00002 & 14 & 60 & 0.1 & 10 \\
\hline 3 & 0 & 24 & 0.4 & 0.4 & 0.00005 & 0.00003 & 0.00002 & 14 & 60 & 0.2 & 12 \\
\hline
\end{tabular}

policy with different objectives[12]. Thus, various combinations of objective function terms and respective penalizing or weighted constants are taken into consideration. Along with these, the initial biomass concentration is also varied. The values of the parameters of the objective function and the initial biomass concentration are given in Table II. 


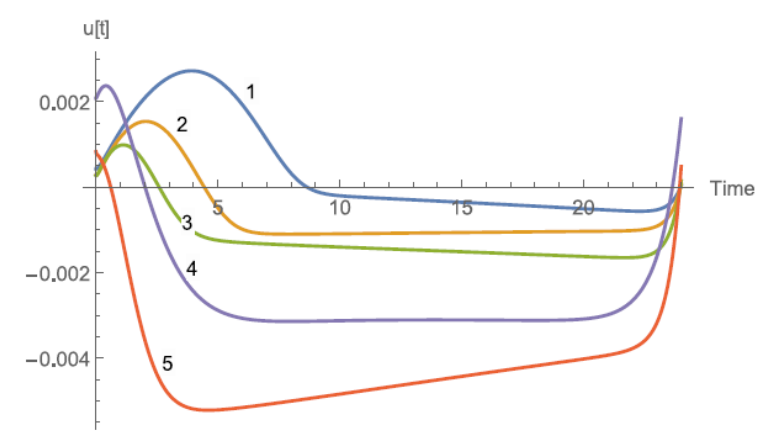

Figure 5: Second numerical experiment. Feed rate control

The cases given in Table 1 were simulated to get an idea of the optimal control feed policy for different variations in the objective function. This is the second numerical experiment. Fig. 5 represents the feed policy for these five cases. For case 1 we observe that no excessive control moves are necessary and bioreaction does not track the product concentration. It is observed that the feed rate is increased in the first few hours and then remains roughly constant to achieve the objectives set by the objective function. In case 2 we see that the objective function considers all the terms except tracking dissolved oxygen concentration. We see a rise in the feed rate a linear decrease throughout and an increase in the ending period.

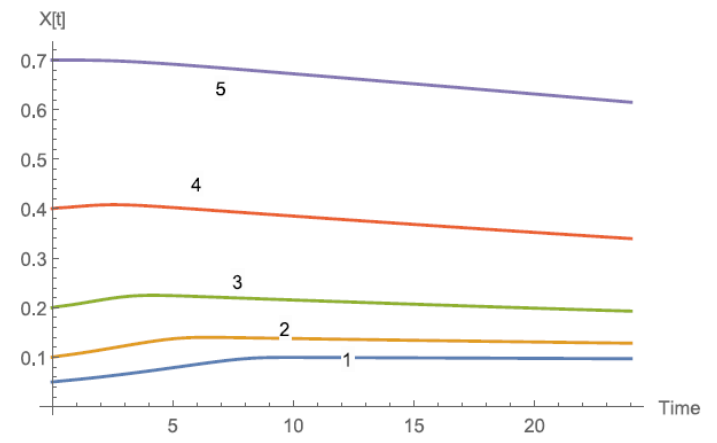

Figure 6: Second numerical experiment. Biomass growth

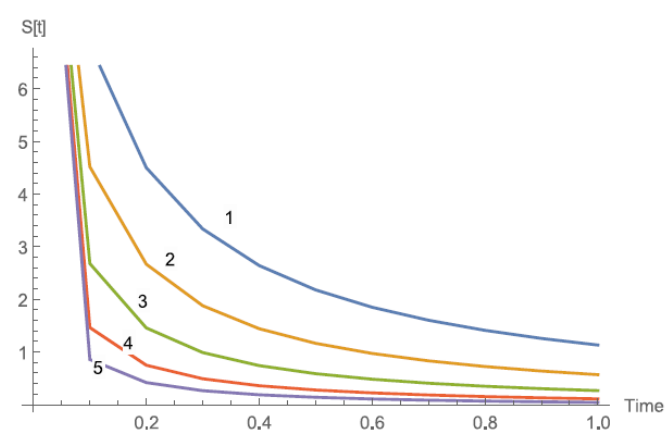

Figure 7: Second numerical experiment. Substrate consumption

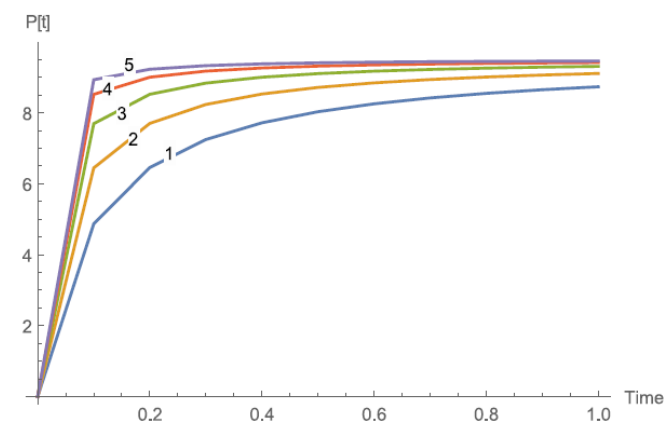

Figure 8: Second numerical experiment. Product formed

Cases 3,4 and 5 show a similar control policy behavior but with differences in values. A higher concentration of biomass brings more death rates to the system thereby forcing the objective function to lower the feed rate so as to maintain the product concentration. This is then followed by a steady rise in the feed rate with a rapid increase in the late period along the batch operation time. Figs. 6, 7, and 8 represent the biomass concentrations, substrate consumption, and product formation respectively. When these cases are studied for substrate consumption, product formation, and biomass concentration in the fed-batch bioreactor during the batch operation time, the results are quite similar to the analysis done in the first part. All these follow similar trends as these parameters depend largely on initial biomass concentration.

\section{Conclusion}

We have successfully done the simulation of a fed batch bioreactor by applying Pontryagin's minimum principle. A gradient method was used to devise the numerical simulation. The optimal feed policy was the result of a simulation experiment.

A parametric study done in this paper resulted in this work giving an insight into how these parameters affect the performance of the system. Future work in this domain can include, using different approaches to solve the optimal control problem or optimization for a fed-batch reaction.

\section{CONFLICT OF INTEREST}

The authors declare no conflict of interest.

\section{ACKNOWLEDGMENT}

Author Dr. Satish Inamdar, thanks to the Department of Science and Technology (DST) for providing research grants to carry out this work.

\section{REFERENCES}

[1] A. D. Rodman, S. Diab, and D. I. Gerogiorgis, "Dynamic Optimization of a Fed-Batch Nosiheptide Reactor," Processes, p. 587, 2020.

[2] D. Bonvin, "Optimal operation of batch reactors - a personal view," Journal of Process Control, vol. 8, no. 5-6, pp. 355-368, 1998.

[3] H. S. Shin and H. C. Lim, "Maximization of metabolite in fed-batch cultures Sufficient conditions for singular arc and optimal feed rate profiles," Biochemical Engineering Journal, vol. 37, no. 1, pp. 6274, 2007. 
[4] S. R. Ponnuswamy, S. L. Shah and C. A. Kiparissides, "Computer optimal control of batch polymerization reactors," Industrial \& Engineering Chemistry Research, pp. 2229-2236, 1987.

[5] Z. K. Nagy and R. D. Braatz, "Worst-Case and Distributional Robustness Analysis of Finite-Time Control Trajectories for Nonlinear Distributed Parameter Systems," IEEE Transactions on Control Systems Technology, vol. 11, no. 5, pp. 694-704, 2003.

[6] J. Zhang, "Batch-to-batch optimal control of a batch polymerization process based on stacked neural network models," Chemical Engineering Science, vol. 63, no. 5, pp. 1273-1281, 2008.

[7] P. M. V. Erdeghem, F. Logist, I. Y. Smets and J. F. V. Impe, "Efficiently solving multiple objective optimal control problems," in The International Federation of Automatic Control, Seoul, 2008.

[8] D. Niu, M. Jia, F. Wang and D. He, "Optimization of nosiheptide fedbatch fermentation process based on a hybrid model," Industrial \& Engineering Chemistry Research, pp. 3373-3380, 2013.

[9] F. Benazet, M. Cartier, J. Florent, C. Godard, G. Jung, J. Lunel, D. Mancy, C. Pascal, J. Renaut, P. Tarridec, J. Theilleux, R. Tissier, M. Dubost, and L. Ninet, "Nosiheptide, a sulfur-containing peptide antibiotic isolated from Streptomyces actuosus 40037," Experientia, vol. 36, no. 4, pp. 414-416, 1980.

[10] A. Locatelli, Optimal Control: An Introduction, Basel; Boston; Berlin: Birkhauser, 2001.

[11] S. A. Senthil and S. Sundaramoorthy, "Optimal control policy for tracking the optimal progression of temperature in a batch reactor-

\section{AUTHORS PROFILE}

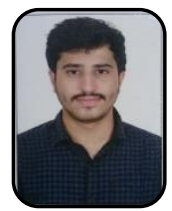

Durgesh Bonde completed his Bachelors in Technology in Chemical Engineering from Vishwakarma Institute of Technology in October 2020. He has been accepted for his Master's in Chemical Engineering at Northeastern University, Boston starting in Fall 2021. He has worked as a Project Intern at the Automotive Research Association of India, Pune in the field of air pollution monitoring and modeling. His research interests are process control, optimization, simulation, sustainable batteries, tissue engineering, energy, and environment, and is looking to work in these fields ahead in his career.

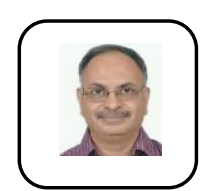

Satish R. Inamdar was born in 1960 . He obtained his M.Tech. in Chemical Engineering and Ph.D. in Chemistry from IIT Bombay and National Chemical Laboratory (registered with the University of Pune) in April 1986 and February 1991 respectively. Later he was a post-doctoral fellow in process control and then a senior project engineer in optimization at the National University of Singapore for five years. $\mathrm{He}$ obtained another Ph.D. Chemical Engineering in January 2014 from National Chemical Laboratory registered with Savitribai Phule Pune University (SPPU). He has about twenty-seven papers published in international journals and about sixty conference papers in International and National conferences. His research interests are nonlinear science, process control, optimization, systems biology, and metamaterials.
Some insights into the choice of an objective function," IFAC Papers Online, vol. 51, no. 1, pp. 112-117, 2018.

[12] I. Y. Smets, D. Dochain and J. F. V. Impe, "Optimal Temperature Control of a Steady-State Exothermic Plug-Flow Reactor," AIChE, vol. 48, pp. 279-286, 2002.

[13] C. V. Peroni, N. S. Kaisare and J. H. Lee, "Optimal Control of a FedBatch Bioreactor Using Simulation-Based Approximate Dynamic Programming," IEEE Transactions on Control Systems Technology, vol. 13 , no. 5, pp. 786-790, 2005.

[14] A. Patkar, J.-H. seo and H. Lim, "Modeling and optimization of cloned invertase expression in Saccharomyces cerevisiae," Biotechnology and Bioengineering, vol. 41, pp. 1066-1074, 1993.

[15] K.-Y. San and G. Stephanopoulos, "Optimization of Fed-batch penicillin fermentation: A Case of Singular Optimal Control with State Constraints," Biotechnology and Bioengineering, vol. 34, pp. 72-78, 1989. 